\title{
THE MODULE OF VECTOR-VALUED MODULAR FORMS IS COHEN-MACAULAY
}

\author{
RICHARD GOTTESMAN
}

\begin{abstract}
Let $H$ denote a finite index subgroup of the modular group $\Gamma$ and let $\rho$ denote a finite-dimensional complex representation of $H$. Let $M(\rho)$ denote the collection of holomorphic vector-valued modular forms for $\rho$ and let $M(H)$ denote the collection of modular forms on $H$. Then $M(\rho)$ is a $\mathbf{Z}$-graded $M(H)$-module. It has been proven that $M(\rho)$ may not be projective as a $M(H)$-module. We prove that $M(\rho)$ is Cohen-Macaulay as a $M(H)$-module. We also explain how to apply this result to prove that if $M(H)$ is a polynomial ring then $M(\rho)$ is a free $M(H)$-module of rank $\operatorname{dim} \rho$.
\end{abstract}

\section{INTRODUCTION}

Let $H$ denote a finite index subgroup of the modular group $\Gamma:=\mathrm{SL}_{2}(\mathbf{Z})$ and let $\rho$ denote a finite-dimensional complex representation of $H$. Let $k \in \mathbf{Z}$ and let $\mathfrak{H}$ denote the complex upper half plane. If $F: \mathfrak{H} \rightarrow \mathbf{C}^{t}$ is a holomorphic function and if $\gamma=\left[\begin{array}{ll}a & b \\ c & d\end{array}\right] \in \Gamma$ then we define $\left.F\right|_{k} \gamma$ by setting $\left.F\right|_{k} \gamma(\tau):=(c \tau+d)^{-k} F\left(\frac{a \tau+b}{c \tau+d}\right)$.

Definition 1.1. A vector-valued modular form $F$ of weight $k$ with respect to $\rho$ is a holomorphic function $F: \mathfrak{H} \rightarrow C^{\text {dim } \rho}$ which is also holomorphic at all of the cusps of $H \backslash\left(\mathfrak{H} \bigcup \mathbb{P}^{1}(\boldsymbol{Q})\right)$ and such that for all $\gamma \in H$,

$$
\left.F\right|_{k} \gamma=\rho(\gamma) F .
$$

The statement that $F$ is holomorphic at all of the cusps of $H \backslash\left(\mathfrak{H} \cup \mathbb{P}^{1}(\mathbf{Q})\right)$ is equivalent to the statement that for each $\gamma \in \Gamma$, each of the component functions of $\left.F\right|_{k} \gamma$ has a holomorphic $q$-expansion. The notion of a holomorphic $q$-expansion is a bit more intricate in the vector-valued setting. A precise treatment of the notion of a holomorphic $q$-expansion can be found for $\Gamma$ in [6] and for an arbitrary subgroup in [9].

The collection of all vector-valued modular forms of weight $k$ for the representation $\rho$ form a finite-dimensional complex vector-space, which we denote by $M_{k}(\rho)$. We let $M_{t}(H)$ denote the collection of all modular forms of weight $t$ on $H$. We define $M(\rho):=\bigoplus_{k \in \mathbf{Z}} M_{k}(\rho)$ and $M(H):=\bigoplus_{k \in \mathbf{Z}} M_{k}(H)$. If $F \in M_{k}(\rho)$ and if $m \in M_{t}(H)$ then $m F \in M_{k+t}(\rho)$. In this way, we view $M(\rho)$ as a $\mathbf{Z}$-graded $M(H)$-module. If $\rho$ is a representation of $\Gamma$ then the module structure of $M(\rho)$ is especially pleasing.

Theorem 1.2. Let $\rho$ denote a representation of $\Gamma$. Then $M(\rho)$ is a free $M(\Gamma)$-module of rank equal to the dimension of $\rho$.

Theorem 1.2 has been used to study the arithmetic of vector-valued modular forms for representations of $\Gamma$ in [10], [5], [12]. There are multiple proofs of Theorem 1.2 and each one offers its own perspective and insights. Theorem 1.2 was proven by Marks and Mason in [11, by Terry Gannon in [7, and by Candelori and Franc in [4]. 
Mason has shown that if $H$ is equal to $\Gamma^{2}$, the unique subgroup of $\Gamma$ of index two, then $M(\rho)$ need not be a free module over $M\left(\Gamma^{2}\right)$. A proof of this fact together with the result that $M(\rho)$ need not even be projective over $M\left(\Gamma^{2}\right)$ appears in Section 6 of [3]. In view of this negative result, it is natural to ask if one may prove a positive result about the structure of $M(\rho)$ as a $M(H)$-module. We prove the following:

Theorem 1.3. $M(\rho)$ is Cohen-Macaulay as a $M(H)$-module.

We shall also explain how to apply Theorem 1.3 to prove the following theorem.

Theorem 1.4. Let $H$ denote a finite index subgroup of $\Gamma$ such that there exist modular forms $X, Y \in M(H)$ for which $X$ and $Y$ are algebraically independent and $M(H)=$ $C[X, Y]$. Then $M(\rho)$ is a free $M(H)$-module of rank dim $\rho$.

We remark that Theorem 1.4 may also be obtained by applying the work of Candelori and Franc in [3]. A complete list of the finitely many subgroups $H$ which satisfy the hypothesis of Theorem 1.4 is given in [1]. Two such subgroups are $\Gamma$ and $\Gamma_{0}(2)$. The author employs Theorem 1.4 to study the arithmetic of vector-valued modular forms on $\Gamma_{0}(2)$ in [9].

In 3], Candelori and Franc study the commutative algebra properties of vector-valued modular forms in a geometric context. If $H$ is a genus zero Fuchsian group of the first kind, with finite covolume and with finitely many cusps, then they define a collection of geometrically weighted vector-valued modular forms, $\operatorname{GM}(\rho)$, which contains $M(\rho)$, and a collection of geometrically weighted modular forms $S(H)$, which contains $M(H)$. They prove that $\operatorname{GM}(\rho)$ is Cohen-Macaulay as a $S(H)$-module. The ideas in [3] involve the classification of vector bundles over orbifold curves of genus zero and are quite interesting. We emphasize that in our paper, Theorem 1.3 applies to the $M(H)$-module $M(\rho)$ and holds for all finite index subgroups of $\Gamma$.

We refer the reader to Benson [2] for the relevant definitions and results from commutative algebra which we shall use in this paper.

\section{AcKnowledgements}

The inspiration for this paper was the proof of the free module theorem in [11]. It is my pleasure to thank Cam Franc, Geoff Mason, Matt Satriano, and Noriko Yui for their interest and encouragement.

\section{PROOFS}

The following lemma will be used in the proof of Theorem 1.3 and Theorem 1.4 .

Lemma 3.1. Let Ind $d_{H}^{\Gamma} \rho$ denote the induction of the representation $\rho$ from $H$ to $\Gamma$. Then $M(\rho)$ and $M\left(\operatorname{Ind} d_{H}^{\Gamma} \rho\right)$ are isomorphic as $\boldsymbol{Z}$-graded $M(\Gamma)$-modules.

Proof. Let $\left\{g_{i}: 1 \leq i \leq[\Gamma: H]\right\}$ denote a complete set of left coset representatives of $H$ in $\Gamma$ where $g_{1}$ denotes the identity matrix. Let $k \in \mathbf{Z}$ and let $F \in M_{k}(\rho)$. We define $\Phi(F):=\left[\left.F\right|_{k} g_{1}^{-1},\left.F\right|_{k} g_{2}^{-1}, \ldots,\left.F\right|_{k} g_{[\Gamma: H]}^{-1}\right]^{T}$, where the superscript $T$ denotes the transpose. We claim that $\Phi(F) \in M_{k}\left(\operatorname{Ind}_{H}^{\Gamma} \rho\right)$. We first note that since $F \in M_{k}(\rho)$, we must have that for all $g \in H$, the function $\left.F\right|_{k} g$ is holomorphic in $\mathfrak{H}$ and it has a holomorphic $q$-expansion. Thus $\Phi(F)$ is holomorphic in $\mathfrak{H}$ and $\Phi(F)$ is holomorphic at the cusp $\Gamma \backslash\left(\mathfrak{H} \bigcup \mathbb{P}^{1}(\mathbf{Q})\right)$ since 
each of its component functions $\left.F\right|_{k} g_{i}^{-1}$ has a holomorphic $q$-expansion. To prove that $\Phi(F) \in M_{k}\left(\operatorname{Ind}_{H}^{\Gamma} \rho\right)$, it now suffices to show that for all $g \in \Gamma$,

$$
\left.\Phi(F)\right|_{k} g=\operatorname{Ind}_{H}^{\Gamma} \rho(g) \Phi(F) .
$$

Let $n=[\Gamma: H]$. Let $\rho^{\bullet}$ denote the function on $\Gamma$ which is defined by the conditions that $\left.\rho^{\bullet}\right|_{H}=\rho$ and $\rho^{\bullet}(g)=0$ if $g \notin H$. With respect to our choice of left coset representatives for $H$ in $\Gamma$, the $i$-th row and $j$-th column block of the matrix $\operatorname{Ind}_{H}^{\Gamma} \rho(g)$ is equal to $\rho^{\bullet}\left(g_{i}^{-1} g g_{j}\right)$. Thus equation 2 is equivalent to the assertion that for each integer $i$ with $1 \leq i \leq n$,

$$
\left.\left(F \mid{ }_{k} g_{i}^{-1}\right)\right|_{k} g=\left.\sum_{t=1}^{n} \rho^{\bullet}\left(g_{i}^{-1} g g_{t}\right) F\right|_{k} g_{t}^{-1} \text {. }
$$

Fix an index $i$ with $1 \leq i \leq n$ and fix $g \in \Gamma$. Then there exists a unique index $j$ for which $g_{i}^{-1} g g_{j} \in H$. We then have that $\left.F\right|_{k} g_{i}^{-1} g g_{j}=\rho\left(g_{i}^{-1} g g_{j}\right) F$. Therefore

$$
\begin{aligned}
\left.\left(\left.F\right|_{k} g_{i}^{-1}\right)\right|_{k} g & =\left.F\right|_{k} g_{i}^{-1} g \\
& =\left.\left(\left.F\right|_{k} g_{i}^{-1} g g_{j}\right)\right|_{k} g_{j}^{-1} \\
& =\left.\left(\rho\left(g_{i}^{-1} g g_{j}\right) F\right)\right|_{k} g_{j}^{-1} \\
& =\left.\rho\left(g_{i}^{-1} g g_{j}\right) F\right|_{k} g_{j}^{-1} \\
& =\left.\sum_{t=1}^{n} \rho^{\bullet}\left(g_{i}^{-1} g g_{t}\right) F\right|_{k} g_{t}^{-1}
\end{aligned}
$$

We have thus proven that equation 3 holds and conclude that $\Phi(F) \in M_{k}\left(\operatorname{Ind}_{H}^{\Gamma}(\rho)\right)$. For each integer $k$, we have defined the map $\Phi: M_{k}(\rho) \rightarrow M_{k}\left(\operatorname{Ind}_{H}^{\Gamma}(\rho)\right)$ and we extend it by linearity to a map $\Phi: M(\rho) \rightarrow M\left(\operatorname{Ind}_{H}^{\Gamma} \rho\right)$.

We now check that $\Phi$ is a map of $\mathbf{Z}$-graded $M(\Gamma)$-modules. Let $m \in M_{t}(\Gamma)$. We recall that $F \in M_{k}(\rho)$. Then

$$
\begin{aligned}
m \Phi(F) & =m\left[\left.F\right|_{k} g_{1}^{-1},\left.F\right|_{k} g_{2}^{-1}, \ldots,\left.F\right|_{k} g_{n}^{-1}\right]^{T} \\
& =\left[\left.m F\right|_{k+t} g_{1}^{-1},\left.m F\right|_{k+t} g_{2}^{-1}, \ldots,\left.m F\right|_{k+t} g_{n}^{-1}\right]^{T} \\
& =\Phi(m F) .
\end{aligned}
$$

Finally, we check that $\Phi$ is a bijection. Let $X \in M_{k}\left(\operatorname{Ind}_{H}^{\Gamma} \rho\right)$. The codomain of $X$ is $\mathbf{C}^{\operatorname{dim}\left(\operatorname{Ind}_{H}^{\Gamma} \rho\right)}$ and $\operatorname{dim}\left(\operatorname{Ind}_{H}^{\Gamma} \rho\right)=n \operatorname{dim} \rho$. Let $X_{1}, \ldots, X_{n}: \mathfrak{H} \rightarrow \mathbf{C}^{\operatorname{dim} \rho}$ denote the holomorphic functions for which $X=\left[X_{1}, \ldots, X_{n}\right]^{T}$. We define the map $\pi$ by setting $\pi(X)=X_{1}$. We will show that $X_{1} \in M_{k}(\rho)$. Let $g \in H$. There exists a unique index $j$ for which $g_{1}^{-1} g g_{j} \in H$. As $g_{1}$ is the identity matrix and $g \in H$, we must have that $g_{j}=g_{1}$. The fact that $X \in M_{k}\left(\operatorname{Ind}_{H}^{\Gamma} \rho\right)$ implies that

$$
\left.X_{1}\right|_{k} g=\sum_{t=1}^{n} \rho^{\bullet}\left(g_{1}^{-1} g g_{t}\right) X_{t}=\rho^{\bullet}\left(g_{1}^{-1} g g_{1}\right) X_{1}=\rho(g) X_{1}
$$

As $X=\left[X_{1}, \ldots, X_{n}\right]^{T}$ is a holomorphic vector-valued modular form, $X_{1}$ is holomorphic in $\mathfrak{H}$ and $X_{1}$ has a holomorphic $q$-expansion. Thus for each $g \in \Gamma,\left.X_{1}\right|_{k} g$ has a holomorphic $q$-expansion since $\left.X_{1}\right|_{k} g=\rho(g) X_{1}$ and $X_{1}$ has a holomorphic $q$-series expansion. We have proven that $X_{1}$ is holomorphic at all of the cusps of $H \backslash\left(\mathfrak{H} \cup \mathbb{P}^{1}(\mathbf{Q})\right)$ and conclude that $\pi(X)=X_{1} \in M_{k}(\rho)$. We also see that $\pi \circ \Phi(F)=\Phi(F)_{1}=\left.F\right|_{k} g_{1}^{-1}=F$ since $g_{1}$ is the identity matrix. As $\pi \circ \Phi$ is equal to the identity map, $\pi$ must be surjective. All that 
remains is to prove that $\pi$ is injective.

Let $X \in M_{k}\left(\operatorname{Ind}_{H}^{\Gamma} \rho\right)$ such that $\pi(X)=0$. We write $X=\left[X_{1}, X_{2}, \ldots, X_{n}\right]^{T}$ where each $X_{i}$ is a holomorphic function from $\mathfrak{H}$ to $\mathbf{C}^{\operatorname{dim} \rho}$. We claim that $X=0$. Suppose not. Then there exists some index $i$ with $X_{i} \neq 0$. Let $g \in g_{i} H g_{1}^{-1}$. Then $g_{i}^{-1} g g_{j} \in H$ if and only if $g_{1}=g_{j}$. Thus $\left.X_{i}\right|_{k} g=\sum_{t=1}^{n} \rho^{\bullet}\left(g_{i}^{-1} g g_{t}\right) X_{t}=\rho\left(g_{i}^{-1} g g_{1}\right) X_{1}$. As $\pi(X)=X_{1}=0$, we have that $\left.X_{i}\right|_{k} g=0$. Hence $X_{i}=\left.\left(\left.X_{i}\right|_{k} g\right)\right|_{k} g^{-1}=0$, a contradiction. Thus $X=0$ and $\pi$ is therefore injective. We have proven that $\pi$ and hence $\Phi$ is a bijection and the lemma now follows.

Let $q=e^{2 \pi i \tau}, \sigma_{3}(n)=\sum_{d \mid n} d^{3}$, and let $S_{k}(\Gamma)$ denote the space of weight $k$ cusp forms on $\Gamma$. We recall the Eisenstein series $E_{4}=1+240 \sum_{n=1}^{\infty} \sigma_{3}(n) q^{n} \in M_{4}(\Gamma)$ and the cusp form $\Delta=q\left(1-q^{n}\right)^{24} \in S_{12}(\Gamma)$.

Lemma 3.2. The sequence $\Delta, E_{4}$ is a regular sequence for the $M(H)$-module $M(\rho)$.

Proof. It is clear that $\Delta$ is a non-zero-divisor for $M(\rho)$ since $\Delta$ has no zeros in $\mathfrak{H}$. To prove that $\Delta$ is regular for $M(\rho)$, it suffices to show that $M(\rho) \neq \Delta M(\rho)$. Suppose that $M(\rho)=\Delta M(\rho)$. Let $X$ denote a non-zero element in $M(\rho)$ of minimal weight $w$. Then $X=\Delta V$ for some $V \in M_{w-12}(\rho)$. The weight of $V$ is less than weight of $X$. This is a contradiction and therefore $M(\rho) \neq \Delta M(\rho)$. We conclude that $\Delta$ is regular for $M(\rho)$.

We will show that $E_{4}$ is regular for $M(\rho) / \Delta M(\rho)$. We have previously shown that $M(\rho) / \Delta M(\rho) \neq 0$. We now argue that $E_{4}$ is a non-zero-divisor for the module $M(\rho) / \Delta M(\rho)$. Suppose that $Y \in M(\rho)$ and $E_{4}(Y+\Delta M(\rho))=\Delta M(\rho)$. Then $E_{4} Y \in \Delta M(\rho)$. We write $E_{4} Y=\Delta Z$ for some $Z \in M(\rho)$. We wish to show that $Y \in \Delta M(\rho)$ and it suffices to prove this when $Y$ is a vector-valued modular form. Let $k$ denote the weight of $Y$. Let $y_{i}$ denote the $i$-th component function of $Y$, let $z_{i}$ denote the $i$-th component function of $Z$, and let $\gamma \in \Gamma$. Therefore $E_{4} y_{i}=\Delta z_{i}$ and $E_{4}\left(\left.y_{i}\right|_{k} \gamma\right)=\Delta\left(\left.z_{i}\right|_{k-8} \gamma\right)$. As $\Delta=q+O\left(q^{2}\right)$ and $E_{4}=1+O(q)$, all the powers of $q$ in $\left.y_{i}\right|_{k} \gamma$ occur to at least the first power. We have thus shown that $\Delta^{-1}\left(\left.y_{i}\right|_{k} \gamma\right)$ contains no negative powers of $q$ and is therefore holomorphic at the cusp $\gamma \cdot \infty$. Hence $\frac{Y}{\Delta}$ is holomorphic at all of the cusps of $H \backslash\left(\mathfrak{H} \cup \mathbb{P}^{1}(\mathbf{Q})\right)$. As $\Delta$ does not vanish in $\mathfrak{H}$, we have that $\frac{Y}{\Delta}$ is holomorphic in $\mathfrak{H}$. Hence $\frac{Y}{\Delta} \in M(\rho)$ and thus $Y+\Delta M(\rho)=\Delta M(\rho)$. We have proven that $E_{4}$ is a non-zero-divisor for the module $M(\rho) / \Delta M(\rho)$.

Finally, we must show that $E_{4}(M(\rho) / \Delta M(\rho)) \neq M(\rho) / \Delta M(\rho)$. We recall that $X$ denotes a nonzero element in $M(\rho)$ of minimal weight $w$. If $M(\rho) / \Delta M(\rho)=E_{4}(M(\rho) / \Delta M(\rho))$ then there exists some $F \in M(\rho)$ such that $X+\Delta M(\rho)=E_{4} F+\Delta M(\rho)$. Let $G \in$ $M(\rho)$ such that $X=E_{4} F+\Delta G$. We may write $F$ and $G$ uniquely as a sum of their homogeneous components. Let $F_{w-4}$ and $G_{w-12}$ denote the weight $w-4$ and the weight $w-12$ homogeneous components of $F$ and $G$. Then $X=E_{4} F_{w-4}+\Delta G_{w-12}$. We must have that $F_{w-4} \neq 0$ or $G_{w-12} \neq 0$ since $X \neq 0$. Thus $F_{w-4}$ or $G_{w-12}$ is a nonzero element of $M(\rho)$ whose weight is less than the weight of $X$. This is a contradiction and we conclude that $M(\rho) / \Delta M(\rho) \neq E_{4}(M(\rho) / \Delta M(\rho))$. We have shown that $E_{4}$ is regular for $M(\rho) / \Delta M(\rho)$ and our proof is complete.

Lemma 3.3. The Krull dimension of the $M(H)$-module $M(\rho)$ is equal to two.

Proof. We recall that the Krull dimension of the $M(H)$-module $M(\rho)$ is defined to be the Krull dimension of the ring $M(H) / \operatorname{Ann}_{M(H)}(M(\rho))$. As the zeros of a nonzero holomorphic function are isolated, $\operatorname{Ann}_{M(H)} M(\rho)=0$. Therefore the Krull dimension of $M(\rho)$ is 
equal to the Krull dimension of $M(H)$. It suffices to prove that the Krull dimension of $M(H)$ is equal to the Krull dimension of $M(\Gamma)=\mathbf{C}\left[E_{4}, E_{6}\right]$, which is equal to two. To do so, we use the fact (see Corollary 1.4.5 in Benson [2]) that if $A \subset B$ are commutative rings and if $B$ is an integral extension of $A$ for which $B$ is finitely generated as an $A$-algebra then the Krull dimensions of $A$ and $B$ are equal. It therefore suffices to show that $M(H)$ is an integral extension of $M(\Gamma)$ and that $M(H)$ is finitely generated as a $M(\Gamma)$-algebra.

Let $\left\{\gamma_{i}: 1 \leq i \leq[\Gamma: H]\right\}$ denote a complete set of right coset representatives of $H$ in $\Gamma$ where $\gamma_{1}$ denotes the identity matrix. If $f \in M_{k}(H)$ then $\left.f\right|_{k} \gamma_{1}=f$ and thus $f$ is a root of the monic polynomial $P(z):=\prod_{i=1}^{[\Gamma: H]}\left(z-\left.f\right|_{k} \gamma_{i}\right) \in M(H)[z]$. If $\gamma \in \Gamma$ then let $\left.P(z)\right|_{k} \gamma$ denote the polynomial obtained by replacing each monomial $c z^{t}$ of $P$ with the monomial $\left(\left.c\right|_{k} \gamma\right) z^{t}$. The fact that $\left\{\gamma_{i} \gamma: 1 \leq i \leq[\Gamma: H]\right\}$ is a complete set of right coset representatives of $H$ in $\Gamma$ together with the fact that $f \in M_{k}(H)$ implies that

$$
\left.P(z)\right|_{k} \gamma=\prod_{i=1}^{[\Gamma: H]}\left(z-\left.f\right|_{k} \gamma_{i} \gamma\right)=\prod_{i=1}^{[\Gamma: H]}\left(z-\left.f\right|_{k} \gamma_{i}\right)=P(z)
$$

Thus $P(z) \in M(\Gamma)[z]$. Hence $M(H)$ is an integral extension of $M(\Gamma)$.

Let $\alpha: H \rightarrow \mathbf{C}^{\times}$denote the trivial representation of $H$. Theorem 1.2 implies that $M\left(\operatorname{Ind}_{H}^{\Gamma} \alpha\right)$ is a free $M(\Gamma)$-module whose rank equals $\operatorname{dim}\left(\operatorname{Ind}_{H}^{\Gamma} \alpha\right)=[\Gamma: H]$. Lemma 3.1 tells us that $M(\alpha)$ and $M\left(\operatorname{Ind}_{H}^{\Gamma} \alpha\right)$ are isomorphic as $M(\Gamma)$-modules. Hence $M(\alpha)=$ $M(H)$ is a free $M(\Gamma)$-module of rank $[\Gamma: H]$. In particular, $M(H)$ is finitely generated as a $M(\Gamma)$-algebra. We conclude that the Krull dimensions of $M(H)$ and $M(\Gamma)$ are equal and the lemma now follows.

We now proceed with the proof of Theorem 1.3.

Proof. We have shown that the Krull dimension of the $M(H)$-module $M(\rho)$ is equal to two and that $M(\rho)$ has a regular sequence of length two. Therefore the depth of $M(\rho)$ is at least two. Moreover, the depth is at most the Krull dimension (see page 50 in [2]), which is equal to two. Hence the depth and the Krull dimension of $M(\rho)$ are both equal to two.

We shall use the following result from commutative algebra to prove Theorem 1.4. This result is stated and proven in Benson's book [2].

Theorem 3.4. [Theorem 4.3.5. in [2]] Let A denote a commutative Noetherian ring and let $M$ denote a finitely generated $A$-module. Assume that $A=\bigoplus_{j=0}^{\infty} A_{j}$ and $M=\bigoplus_{j=-\infty}^{\infty} M_{j}$ are graded, $A_{0}=K$ is a field, and $A$ is finitely generated over $K$ by elements of positive degree. Then the following statements are equivalent:

(i): $M$ is Cohen-Macaulay.

(ii): If $x_{1}, \ldots x_{n} \in A$ are homogenous elements generating a polynomial subring

$K\left[x_{1}, \ldots, x_{n}\right] \subset A / A n n_{A}(M)$, over which $M$ is finitely generated, then $M$ is a free $K\left[x_{1}, \ldots, x_{n}\right]$-module.

We now give the proof of Theorem 1.4.

Proof. We first note that the hypotheses of Theorem 3.4 are satisfied if we take $A=M(H)$ and $M=M(\rho)$. Thus statements (i) and (ii) in Theorem 3.4 are equivalent if $A=M(H)$ and $M=M(\rho)$. We have proven that $M(\rho)$ is Cohen-Macaulay as a $M(H)$-module. 
Thus statement (i) and hence statement (ii) in Theorem 3.4 must be true. In particular, if $X, Y \in M(H)$ which are algebraically independent then $M(\rho)$ is a free $\mathbf{C}[X, Y]$-module. The hypothesis of Theorem 1.4 asserts that there exist such modular forms $X$ and $Y$ for which $M(H)=\mathbf{C}[X, Y]$. Thus the hypothesis of Theorem 1.4 implies that $M(\rho)$ is a free $M(H)$-module.

We now compute the rank $r$ of $M(\rho)$ as a $M(H)$-module. We have have shown in the proof of Lemma 3.3 that $M(H)$ is a free $M(\Gamma)$-module of rank $[\Gamma: H]$. Therefore $M(\rho)$ is a free $M(\Gamma)$-module of rank $[\Gamma: H] r$. Theorem 1.2 tells us that $M\left(\operatorname{Ind}_{H}^{\Gamma} \rho\right)$ is a free $M(\Gamma)$ module whose rank equals $\operatorname{dim}\left(\operatorname{Ind}_{H}^{\Gamma} \rho\right)=[\Gamma: H] \operatorname{dim} \rho$. Lemma 3.1 states that $M(\rho)$ and $M\left(\operatorname{Ind}_{H}^{\Gamma} \rho\right)$ are isomorphic as $M(\Gamma)$-modules. Thus $M(\rho)$ is a free $M(\Gamma)$-module whose rank equals $[\Gamma: H] \operatorname{dim} \rho$. Hence $[\Gamma: H] r=[\Gamma: H] \operatorname{dim} \rho$ and $r=\operatorname{dim} \rho$.

\section{REFERENCES}

[1] Eiichi Bannai, Masao Koike, Akihiro Munemasa, and Jiro Sekiguchi. Some results on modular forms - subgroups of the modular group whose ring of modular forms is a polynomial ring. In Groups and combinatorics - in memory of Michio Suzuki, volume 32 of Adv. Stud. Pure Math., pages 245254. Math. Soc. Japan, Tokyo, 2001.

[2] David J. Benson. Polynomial invariants of finite groups, volume 190. Cambridge University Press, 1993.

[3] Luca Candelori and Cameron Franc. Vector bundles and modular forms for Fuchsian groups of genus zero. arXiv preprint arXiv:1704.01684, 2017.

[4] Luca Candelori and Cameron Franc. Vector-valued modular forms and the modular orbifold of elliptic curves. International Journal of Number Theory, 13(01):39-63, 2017.

[5] Cameron Franc and Geoffrey Mason. Fourier coefficients of vector-valued modular forms of dimension 2. Canadian Mathematical Bulletin, 57(3):485-494, 2014.

[6] Cameron Franc and Geoffrey Mason. Hypergeometric series, modular linear differential equations and vector-valued modular forms. The Ramanujan Journal, 41(1-3):233-267, 2016.

[7] Terry Gannon. The theory of vector-valued modular forms for the modular group. In Conformal Field Theory, Automorphic Forms and Related Topics, pages 247-286. Springer, 2014.

[8] Richard Gottesman. The algebra and arithmetic of vector-valued modular forms on $\Gamma_{0}(2)$. $\mathrm{PhD}$ thesis, University of California, Santa Cruz, 2018.

[9] Richard Gottesman. The arithmetic of vector-valued modular forms on $\Gamma_{0}(2)$. arXiv preprint arXiv:1811.04452, 2018.

[10] Christopher Marks. Fourier coefficients of three-dimensional vector-valued modular forms. Commun. Number Theory Phys., 9(2):387-412, 2015.

[11] Christopher Marks and Geoffrey Mason. Structure of the module of vector-valued modular forms. Journal of the London Mathematical Society, 82(1):32-48, 2010.

[12] Geoffrey Mason. On the Fourier coefficients of 2-dimensional vector-valued modular forms. Proceedings of the American Mathematical Society, 140(6):1921-1930, 2012.

Department of Mathematics and Statistics, Queen's University, Kingston, ON K7L3N6 E-mail address: richard.b.gottesman@gmail.com 